\title{
Nonlinear continuous-time generalized predictive control of solar power plant
}

\author{
Billal Khoukhi*, Mohamed Tadjine, and Mohamed Seghir Boucherit \\ Process Control Laboratory, Control Engineering Department, École Nationale Polytechnique, 10 avenue Pasteur, \\ Hassan-Badi, 16131 Algiers, Algeria
}

Received 3 April 2015 / Accepted 30 May 2015

\begin{abstract}
This paper presents an application of nonlinear continuous-time generalized predictive control (GPC) to the distributed collector field of a solar power plant. The major characteristic of a solar power plant is that the primary energy source, solar radiation, cannot be manipulated. Solar radiation varies throughout the day, causing changes in plant dynamics and strong perturbations in the process. A brief description of the solar power plant and its simulator is given. After that, basic concepts of predictive control and continuous-time generalized predictive control are introduced. A new control strategy, named nonlinear continuous-time generalized predictive control (NCGPC), is then derived to control the process. The simulation results show that the NCGPC gives a greater flexibility to achieve performance goals and better perturbation rejection than classical control.
\end{abstract}

Key words: Predictive control, Solar power plant, Nonlinear system description.

\section{Introduction}

In recent years, the use of renewable energies for reducing environmental impact of fossil energies has grown considerably. There is a pressing need to find inexhaustible energy sources. Solar energy is by far the most abundant energy source of renewable energy. One of the main characteristics of a solar power plant is that the primary energy source (solar radiation) cannot be manipulated. Besides, the solar radiation intensity depends on daily and seasonal cycle variations, like clouds, atmospheric humidity, and air transparency. This justifies the relevance of solar power plant control. The distributed collector field consists of a series of parabolic mirrors that reflect solar radiation on a pipe where oil gets heated while circulating. The objective of the control system in a distributed collector field is to maintain the outlet oil temperature at a desired level in spite of disturbances, such as changes in the solar irradiance level (caused by clouds), mirrors' reflectance, or inlet oil temperature. This is accomplished by varying the flow of the fluid through the field. The field exhibits a variable delay time that depends on the control variable (flow). The transfer function of the process varies with factors such as irradiance level, mirrors' reflectance, and oil inlet temperature. Those changes affect the dynamics of the process.

*Corresponding author: khoukhi.billal@gmail.com
In order to reduce the impact of disturbances, a feed-forward controller is needed [1].

Control of solar plants has been addressed in many ways. In Igreja et al. [2], a nonlinear control using exact feedback linearization and Lyapunov's approach is presented. In Camacho et al. [3], a survey on advanced control strategies tested in the Acurex experimental plant at the PSA (Solar Plant of Almería in Spain) is described. In Limon et al. [4], a robust tracking model predictive control (MPC) is presented. In Garcia-Gabin et al. [5], a sliding model predictive control is developed. In Gallego \& Camacho [6], an adaptive state-space model predictive control is presented.

This paper applies nonlinear continuous-time model predictive control into solar power plants. The main benefit of the proposed technique is to achieve optimal performance as defined by a performance index without the need of on-line optimization that is normally required for implementing a model predictive control strategy. The on-line nonlinear optimization imposes a heavy computational burden, which requires extensive computing power and a long sampling time in engineering implementation, this is why it is natural to develop a generalized predictive control (GPC) in a continuous-time setting by pursuing an analytic approach, where it is intended to develop a closed form nonlinear model predictive control (NMPC) where on-line optimization is not required. 


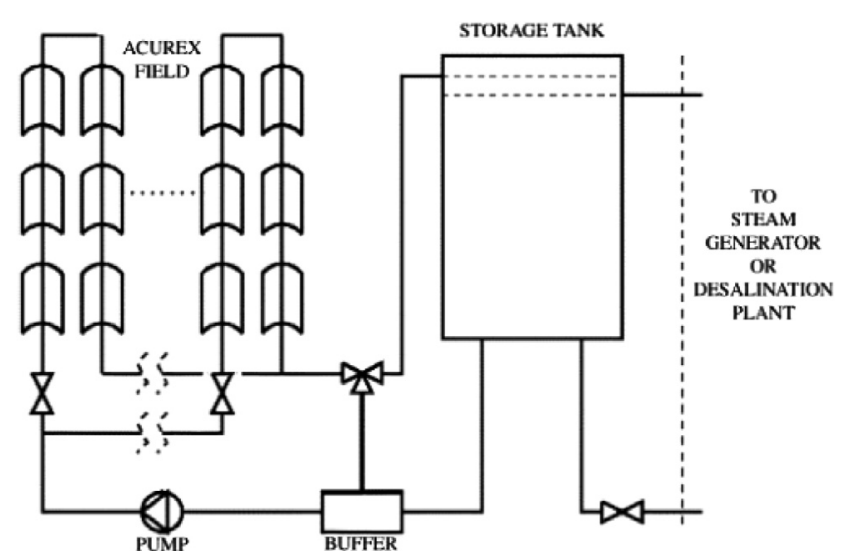

Figure 1. Schematic diagram of the Acurex distributed collector field.

The paper is organized as follows: Section 2 describes the mathematical models of a collector loop. In Section 3, the new NCGPC algorithm and the obtaining of the control law are developed. In Section 4, simulations and results are described. Finally, the paper draws to a close with some concluding remarks.

\section{Solar power plant}

\subsection{Process description}

The main objective of the solar plant is to use solar radiation to heat oil passing through the field. The field consists of collectors that are arranged in rows that form parallel loops. The inlet oil is extracted from the bottom of a storage tank and is passed through the field using a pump located in the field inlet. This fluid is heated and then introduced back into the storage tank to be used for electricity generation.

The considered solar power plant is located in Almería, Spain, which is a tested and validated model of a trough collector loop [1]. As shown in Figure 1, the Acurex field consists of 480 collectors arranged in 20 rows that form 10 parallel loops and lie along an east-west axis [3].

\subsection{Mathematical model}

In the case of control applications, the most important consideration is to describe the outlet oil temperature as a function of the flow rate of the oil. Generally, such a model could be formed by applying the energy conservation law for a length control volume of $\mathrm{d} l$ in the collector over a time interval $\mathrm{d} t$. The actual equation can be set up as:

$\rho_{f} C_{f} A_{f} \frac{\partial T_{f}}{\partial t}+\rho_{f} C_{f} q \frac{\partial T_{f}}{\partial l}=I G n o K_{\mathrm{opt}}-H_{l} G\left(T_{f}-T_{a}\right)$

where $f$ the subscript refers to the oil fluid. Also $t$ is the time $(\mathrm{s}) ; x$ is the position $(\mathrm{m}) ; \rho$ is the oil density $\left(\mathrm{kg} / \mathrm{m}^{3}\right) ; c$ is the specific heat of oil $(\mathrm{J} / \mathrm{kgK}) ; A$ is the cross-section of the pipeline $\left(\mathrm{m}^{2}\right) ; T$ is the oil temperature $\left({ }^{\circ} \mathrm{C}\right) ; I$ is the solar radiation; no is the geometric efficiency; $H_{l}$ is the global

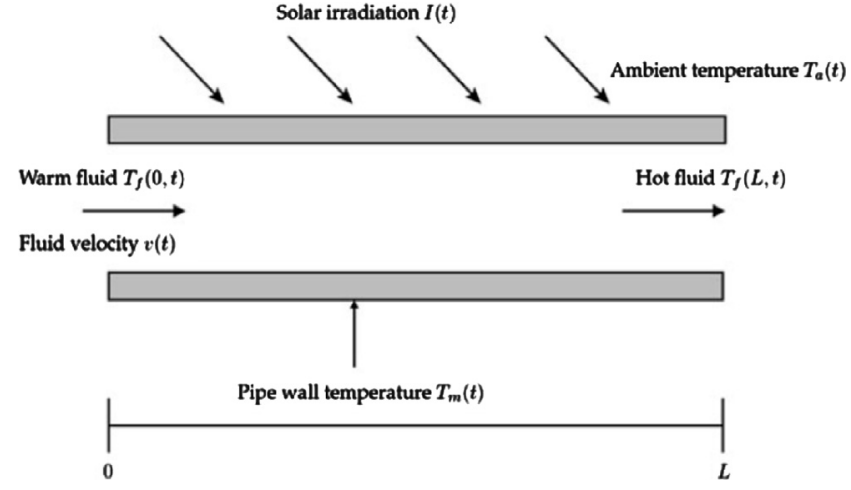

Figure 2. Parabolic trough cross-section.

coefficient of thermal losses $\left(\mathrm{W} / \mathrm{m}^{2}{ }^{\circ} \mathrm{C}\right) ; G$ is the collector aperture $(\mathrm{m}) ; T_{a}$ is the environment temperature $\mathrm{C} ; q$ is the oil flow rate $\left(\mathrm{m}^{3} / \mathrm{s}\right) ; K_{\mathrm{opt}}$ is the optical efficiency.

Parameters $\rho_{f}$ and $c_{f}$ are function of temperature and so function of time and position.

Notice that the partial differential equation represented in equation (1) when discretized in space the following expression is obtained:

$$
\begin{aligned}
\frac{\mathrm{d} T_{f}(n, t)}{\mathrm{d} t}= & \frac{1}{\rho_{f}(n) C_{f}(n) A_{f}}\left(\text { InoGK }_{\mathrm{opt}}\right. \\
& \left.-H_{l}(n) G\left(T_{f}(n, t)-T_{a}\right)\right)-\frac{q}{A_{f} \Delta l} \\
& \times\left(T_{f}(n, t)-T_{f}(n-1, t)\right)
\end{aligned}
$$

where $n$ is the $n$th segment in which the pipe is divided. The system has four inputs (the ambient temperature $T_{a}$, the effective solar radiation InoK $_{\text {opt }}$, the inlet temperature $T(0, t)$, and the oil flow $q)$ and one output (the outlet temperature $T(N, t))$ where $N$ is the last segment that the pipe is divided. Figure 2 shows the schematic of discretization of the parabolic trough cross-section.

Let us put $T(n, t)=x_{n}$, so that the output of the plant is then $Y=x_{N}=T(N, t)$ and the inputs are: $U_{q}=q, U_{T a}=T_{a}$, $U_{\text {irr }}=I G n o K_{\text {opt }}$, and $U_{\text {Tin }}=x_{0}=T(0, t)$. We can then write:

$$
\begin{gathered}
\dot{x}_{1}=\frac{1}{\rho_{f}(1) C_{f}(1) A_{f}}\left(\mathrm{U}_{\mathrm{irr}}-H_{l}(1) G\left(x_{1}-U_{T a}\right)\right)-\frac{U_{q}}{A_{f} \Delta l}\left(x_{1}-x_{0}\right) \\
\dot{x}_{2}=\frac{1}{\rho_{f}(2) C_{f}(2) A_{f}}\left(\mathrm{U}_{\mathrm{irr}}-H_{l}(2) G\left(x_{2}-U_{T a}\right)\right)-\frac{U_{q}}{A_{f} \Delta l}\left(x_{2}-x_{1}\right) \\
\vdots \\
\dot{x}_{N}=\frac{1}{\rho_{f}(\mathrm{~N}) C_{f}(\mathrm{~N}) A_{f}}\left(\mathrm{U}_{\mathrm{irr}}-H_{l}(\mathrm{~N}) G\left(x_{N}-U_{T a}\right)\right)-\frac{U_{q}}{A_{f} \Delta l}\left(x_{N}-x_{N-1}\right) .
\end{gathered}
$$

The control objective is to maintain the outlet temperature $Y=x_{N}=T(N, t)$ at constant value using the oil flow $U_{q}=q$. As explained previously, the plant has many control difficulties: (1) the main source of energy (solar irradiation) cannot be manipulated. (2) The solar irradiation is a fast perturbation that acts directly to the output. (3) The system is highly nonlinear mainly at high temperatures of the oil. This is why a classical control law cannot deal with these difficulties, and an advanced control is necessary to encounter those difficulties. 


\section{Nonlinear generalized predictive control}

Model predictive control (MPC) is a control strategy which uses a mathematical model of the plant to predict its evolution and, taking into account this evolution, find the control signal which minimizes a cost function [8].

Various nonlinear predictive control methods for discretetime systems have been developed. The main shortcoming of those methods is that on-line dynamic optimization is required, which, in general, is non-convex, and its computational burden grows exponentially with the decision variables. Hence, computational issue is an active subject in predictive control. There are several model predictive controllers implemented in the Acurex field. For example, in Silva et al. [9] a variable sample time statespace adaptive controller is proposed. In Igreja et al. [10], a state-space receding horizon controller, which explicitly explores the distributed parameter character of the solar field, is developed.

Solar power plant is described by continuous-time differential equations and it is natural to develop GPC in a continuoustime setting. The interest in developing a continuous-time predictive control in nonlinear environment is that the on-line optimization involved in NMPC is non-convex, which implies that the optimization procedure might be terminated at a local minimum. Consequently, poor performance even instability might result after the control sequence yielded by the on-line optimizer is implemented [7]. The heavy computing power needed in the on-line optimization requires to develop GPC in a continuous-time setting by pursuing an analytic approach.

The NCGPC algorithm solves those constraints and has been applied several times for other different systems as in Chen [12]. Alike, this paper uses the advantages of this control law to achieve optimal performance of solar power plants. However, to apply the NCGPC strategy, the following assumptions should be respected $[11,12]$ :

- the dynamic of zeros exists and is asymptotically stable,

- all stats are measurable,

- the relative degree $\rho$ is defined,

- the output $y(t)$ and the reference signal are continuously differentiable in time [13].

Once the assumptions are verified we could start the development of the output. In the NCGPC algorithm the prediction of the output is given by its development of Taylor series:

$$
\hat{y}(t+\tau)=\sum_{k=0}^{\rho} y^{(k)}(t) \frac{\tau^{k}}{k !}+R\left(\tau^{\rho}\right)
$$

where $t$ represents the present time, $t+\tau$ represents the prediction instant, $\rho$ represents the relative degree of the plant, and $R\left(\tau^{\rho}\right)$ is the high order element when neglected (3) becomes:

$$
\hat{y}(t+\tau)=\sum_{k=0}^{\rho} y^{(k)}(t) \frac{\tau^{k}}{k !} .
$$

In the same way as (4) the prediction of the reference is obtained:

$$
\hat{\omega}(t+\tau)=\sum_{k=0}^{\rho} \omega^{(k)}(t) \frac{\tau^{k}}{k !}
$$

The objective is to find a control law that makes the tracking error tend to zero when $t$ tends to $\infty$. The prediction error is defined as:

$$
\hat{e}(t+\tau)=\hat{y}(t+\tau)-\hat{\omega}(t+\tau) .
$$

The cost function is then given as:

$$
J(t)=\frac{1}{2} \int_{0}^{T} \hat{e}(t+\tau)^{2} \mathrm{~d} \tau .
$$

Theorem: Consider the solar plant model (2) and the performance criterion $J$ (7). Then, the control law $U_{q}$ given in equation (8) minimizes $J$.

$$
\begin{aligned}
U_{q}= & \frac{3}{2 T} \frac{A_{f} \Delta l\left(x_{7}-\omega\right)-A_{f} \Delta l \dot{\omega}}{T(\mathrm{~N})-T(N-1)} \\
& +\frac{A_{f} \Delta l\left(I-H_{l} G\left(T(N)-T_{a}\right)\right)}{\rho_{f} A_{f} C_{f}(T(\mathrm{~N})-T(N-1))} .
\end{aligned}
$$

Proof:

The output in a solar power plant is the outlet temperature that represents the last segment that the pipe is divided; its derivative is obtained by subtracting $n$ by $N$ in equation (2):

$$
\begin{aligned}
\dot{y}= & \frac{1}{\rho_{f}(N) C_{f}(N) A_{f}}\left(\operatorname{Ino} G K_{\mathrm{opt}}-H_{l}(N) G\left(y-T_{a}\right)\right) \\
& -\frac{q}{A_{f} \Delta l}\left(\mathrm{y}-T_{f}(\mathrm{~N}-1, t)\right) .
\end{aligned}
$$

The relative degree of the plant of the plant is $\rho=1$. By subtracting $\rho$ by its values in equations (4) and (5):

$$
\begin{gathered}
y(t+\tau)=y+\tau \dot{y}=\left[\begin{array}{ll}
1 & \tau
\end{array}\right]\left[\begin{array}{l}
y \\
\dot{y}
\end{array}\right] \\
\omega(t+\tau)=\omega+\tau \dot{\omega}=\left[\begin{array}{ll}
1 & \tau
\end{array}\right]\left[\begin{array}{c}
\omega \\
\dot{\omega}
\end{array}\right] .
\end{gathered}
$$

The tracking error is defined as the difference between the output and the reference signal:

$$
e(t)=y(t)-\omega(t) .
$$

Let us define vectors $\Lambda(\tau), Y(t), \Omega(t)$ so that:

$$
\Lambda(\tau)=\left[\begin{array}{ll}
1 & \tau
\end{array}\right], Y(t)=\left[\begin{array}{l}
y(t) \\
\dot{y}(t)
\end{array}\right], \Omega(t)=\left[\begin{array}{c}
\omega(t) \\
\dot{\omega}(t)
\end{array}\right] .
$$

Let us define another vector $E(t)$ so that:

$$
E(t)=Y(t)-\Omega(t)
$$


When replacing in equation (7):

$$
\hat{e}(t+\tau)=\Lambda(\tau) Y(t)-\Lambda(\tau) \Omega(t) .
$$

And then:

$$
\hat{e}(t+\tau)=\Lambda(\tau) E(t) .
$$

The formula to express the cost function can be obtained.

When replacing $\hat{e}(t+\tau)$ in the criterion by its expression given in equation (10):

$$
J(t)=\frac{1}{2} \int_{0}^{T} E(t)^{T} \Lambda(\tau)^{T} \Lambda(\tau) E(t) \mathrm{d} \tau
$$

and then,

$$
J(t)=\frac{1}{2} E(t)^{T}\left[\int_{0}^{T} E(t)^{T} \Lambda(\tau)^{T} \Lambda(\tau) \mathrm{d} \tau\right] E(t)
$$

with:

$$
\int_{0}^{T} E(t)^{T} \Lambda(\tau)^{T} \Lambda(\tau) \mathrm{d} \tau=\left[\begin{array}{cc}
T & \frac{T^{2}}{2} \\
\frac{T^{2}}{2} & \frac{T^{3}}{3}
\end{array}\right] .
$$

The control law can be obtained by minimizing the cost function (7):

$$
\frac{\partial J}{\partial u}=0
$$

and then,

$$
\left(\frac{\partial E(t)}{\partial u}\right)^{T}\left[\begin{array}{cc}
T & \frac{T^{2}}{2} \\
\frac{T^{2}}{2} & \frac{T^{3}}{3}
\end{array}\right] E(t)=0 .
$$

The output of the NCGPC controller can be found by resolving the equation (11):

$$
\begin{aligned}
U_{q}= & \frac{3}{2 T} \frac{A_{f} \Delta l\left(x_{7}-\omega\right)-A_{f} \Delta l \dot{\omega}}{T(\mathrm{~N})-T(N-1)} \\
& +\frac{A_{f} \Delta l\left(I-H_{l} G\left(T(N)-T_{a}\right)\right)}{\rho_{f} A_{f} C_{f}(T(\mathrm{~N})-T(N-1))} .
\end{aligned}
$$

When reference signal $x(t)$ is canceled, the control law is the input-output linearization (FL) without poles placement terms. For SISO nonlinear systems, when the relative degree is almost equal to 4, the closed loop stability is ensured [12]. For $\omega(t)$ equal to zero, the control law (12) is exactly that of the FL [16]. The only difference is that the coefficients $K$ that
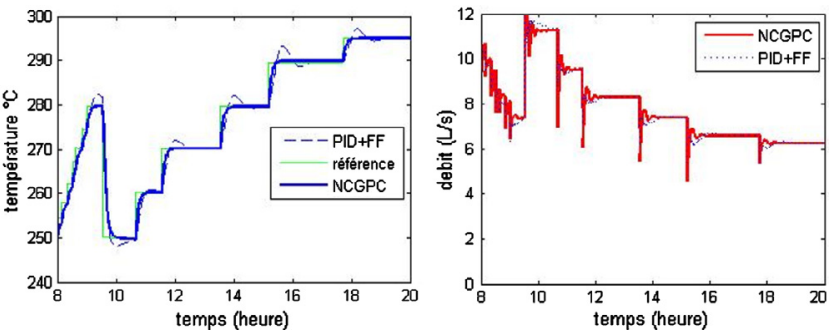

Figure 3. NCGPC response compared with PID + feed forward.

characterize the stability of the closed loop system are different from those of the FL. For NCGPC they result directly from the minimization of the cost function, whereas in FL, they are arbitrarily chosen to ensure the closed loop stability. The NCGPC is then, an optimal FL in a new coordinate system [17].

\section{Simulation results}

\subsection{NCGPC response}

Due to the degree of nonlinearities, linear methods cannot perform well in this plant under all operation conditions, as can be seen in Figure 3, the fixed parameters PID with feedforward control developed in Camacho et al. [14] cannot deal with the high nonlinearities at high temperature.

The NCGPC developed deals well with these nonlinearities and possesses a lower time response, this is due to the predictive and the nonlinear characteristic of the control law.

\subsection{Influence of prediction horizon}

To study the influence of the prediction horizon let us take the general form of first-order system similar to our system:

$$
z_{1}=h-\omega .
$$

The resulting system is given by:

$$
\dot{z}_{1}=L_{f} h-\dot{\omega}+u L_{g} h .
$$
have:

By replacing $u$ by the control law given in equation (12) we

$$
\dot{z}_{1}=-k_{10} z_{1}
$$

with:

$$
k_{10}=\frac{3}{2 T} .
$$

We can then obtain the characteristic polynom of the closed loop system:

$$
P_{1 T}(\lambda)=k_{10}+\lambda .
$$



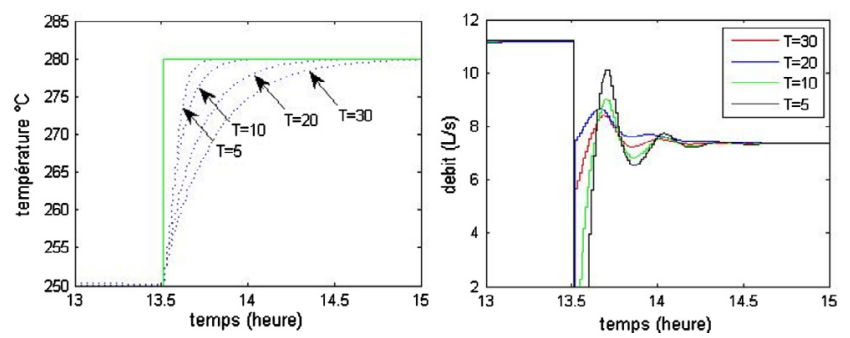

Figure 4. Influence of the prediction horizon in NCGPC.

Let us consider $D_{1}(p)$ the denominator of the first-order transfer function $H_{1}(p)$ :

$$
H_{1}(p)=\frac{G_{1}}{1+\theta p}
$$

$G_{1}$ and $\theta$ represent the static gain and the time constant, respectively. Then $D_{1}(p)=1+\theta p$.

The transfer function $H_{1}(p)$ has a unique pole $p=-1 / \theta$. On the other hand the polynomial $P_{1 T}(\lambda)$ has unique root $\lambda=-K_{10}$. By equalizing those two equations we obtain:

$$
k_{10}=\frac{1}{\theta}
$$

But $K_{10}=3 / 2 T=1 / \theta$. And so:

$$
T=\frac{3 \theta}{2}
$$

We can conclude that the response time is function of the prediction horizon:

$$
t_{r}=3 \theta=2 T \text {. }
$$

When $T$ increases the root $\lambda=-3 / 2 \mathrm{~T}$ tends to zero but remains in the negative side of the complex plan, and the response time increases linearly. The stability is then guaranteed and the time response is set by manipulating the prediction horizon. To illustrate this result we have simulated the influence of the prediction horizon in Figure 4.

As demonstrated, by increasing the prediction horizon, the response time increases. We can notice also, that the control signal is more energetic when the control horizon is small; we should then determine a compromise between the response time and constraints.

\subsection{Robustness tests}

To ensure that the control law gives proper results, robustness tests are conducted. The two main different disturbances affecting the robustness of the solar power plant system are the change in system parameters and the solar irradiation.

The change in system parameters is due to the synthetic oil used in the system. The characteristic of the synthetic oil used
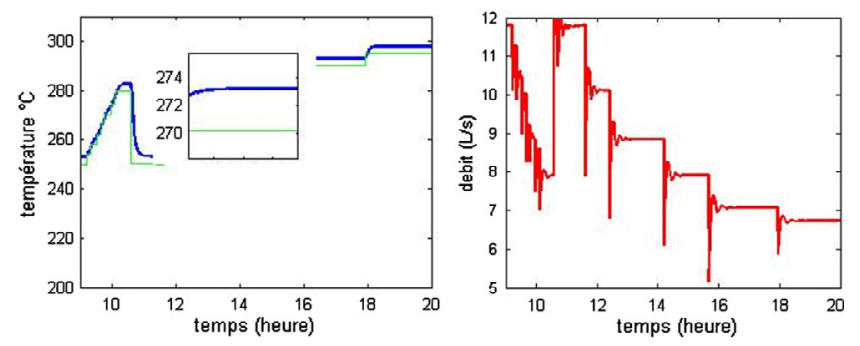

Figure 5. Response of NCGPC with parameter variations.
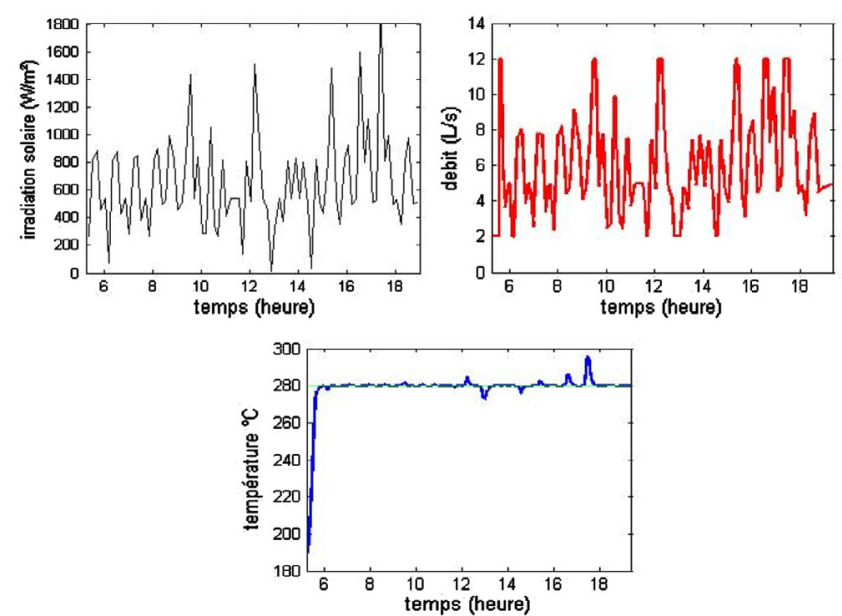

Figure 6. Response of NCGPC to the irradiation of the 04/05/2013 at Ouergla (Algeria).

in the solar power plant can change during the entire heating process. Also, parameters $\rho_{f}$ and $C_{f}$ can change with time and so influence the response of the entire plant. It is necessary that the control law developed deals with those variations. Figure 5 shows the influence of a variation of $5 \%$ of those parameters.

As can be seen, the variation introduced affects the response of the control law by acting directly on the outlet temperature, this can be seen by a deviation from the reference signal, this deviation is too small to be taken into account, and the response can be considered as satisfying.

As explained, the second test deals with outside perturbation. The solar irradiation is the main perturbation; it can directly affect the outlet temperature and acts with high frequency in an unpredictable way. In order to simulate a fast variation of this perturbation, we have used a model [15] of solar irradiation to simulate the day of 04/05/2013 at Ouargla (Algeria) city. The results of this simulation are given in Figure 6.

As can be seen, the very strong variation of the solar irradiation of that day directly affects the outlet temperature, the NCGPC reacts correctly to this variation by adjusting the oil flow, and this makes the tracking process work well with small deviation in the presence of the strong variation of the irradiation. This response makes the NCGPC a robust controller that deals correctly with perturbations and parameter variations. 


\section{Conclusion}

In this paper a nonlinear continuous-time generalized predictive controller has been developed. The simulations, which have been carried out, demonstrate that the performances of the controller are very good in most cases. The NCGPC controller surpasses the performance of finetuned PID + feed forward. The control law shows its optimality due to its cost function minimization and its robustness due to the use of the real output for predicting the future output. The optimality is, however, approximate due to the approximating system dynamic with its Taylor expansion. The continuous characteristic of the control law developed allowed resolving the computational issue and the non-convex optimization. In general, the outcome can be considered satisfactory.

\section{References}

1. Camacho EF, Berenguel M, Rubio FR. 1997. Advanced control of solar plant. Springer-Verlag: London, UK.

2. Igreja JM, Lemos JM, Barao M, Silver RN. 2003. Adaptive nonlinear control of a distributed collector solar field, in Proc. of European Control Conference 2003, ECC03.

3. Camacho EF, Rubio F, Berenguel M, Valenzuela L. 2007. A survey on control schemes for distributed solar collector fields. Part I: modeling and basic control approaches. Solar Energy, 81, 1240-1251.

4. Limon D, Alvarado I, Alamo T, Ruiz M, Camacho EF. 2008. Robust control of the distributed solar collector field ACUREX using MPC for tracking, in Proceedings of 17th IFAC World Congress, Seoul, Korea, Vol. 17, p. 958-963.

5. Garcia-Gabin W, Zambrano D, Camacho EF. 2009. Sliding mode predictive control of a solar air conditioning plant. Control Engineering Practice, 17, 652-663.
6. Gallego AJ, Camacho EF. 2012. Adaptative state-space model predictive control of a parabolic-trough field. Control Engineering Practice, 20, 904-911.

7. Chen W, Ballance DJ, Gawthrop PJ. 1999. Analytic approach to generalized predictive control of nonlinear systems. IEE: Savoy Place, London WCPR OBI-, UK.

8. Camacho EF, Bordons C. 2004. Model predictive control, 2nd edition. Springer Verlag: London. DOI: 10.1007/978-0-85729398-5.

9. Silva RN, Lemos JM, Rato LM. 2003. Variable sampling adaptive control of a distributed collector solar field. IEEE Transactions on Control Systems Technology, 5(11), $765-771$

10. Igreja J, Lemos J, Silva J. 2005. Adaptative receding horizon control of a distributed solar collector field, in Proceedings of the 44th IEEE conference on decision and control, and the European control conference. p. 1282-1287. DOI: 10.1109/ CDC.2005.1582335.

11. Chen WH. 2001. Analytic predictive controllers for nonlinear systems with ill-defined relative degree. IEE Proceedings Control Theory and Applications, 148(1), 9-16, ISSN: $1350-2379$.

12. Chen WH. 2003. Optimal control of nonlinear systems: a predictive control approach. Automatica, 39(1), 633-641.

13. Nijmeijer H, van der Schaft AJ. 1990. Nonlinear dynamical control systems. Springer Verlag: New York.

14. Camacho EF, Berenguel M, Rubio FR, Martínez D. 2012. Control of solar energy systems, in Advances in industrial control. Springer-Verlag London Limited. DOI: 10.1007/978-085729-916-1_2.

15. ASHRAE. 1999. ASHRAE handbook: HVAC applications. Atlanta (GA).

16. Isidori A. 1995. Nonlinear control systems, 3rd edition. Springer-Verlag, Englewood Cliffs: New York.

17. Dabo M, Chafouk H. 2010. Commande prédictive non linéaire a temps continu de systèmes complexes. Editions universitaires européennes. ISBN-13: 978-613-1-54339-5.

Cite this article as: Khoukhi B, Tadjine $M \&$ Boucherit MS: Nonlinear continuous-time generalized predictive control of solar power plant. Int. J. Simul. Multisci. Des. Optim., 2015, 6, A3. 